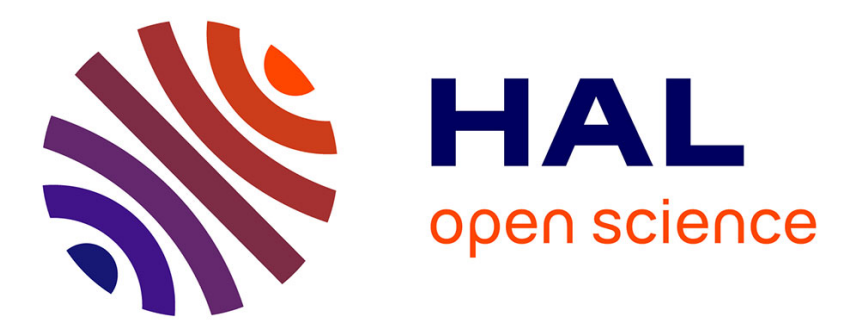

\title{
A Cascades Approach to Formation-Tracking Stabilization of Force-Controlled Autonomous Vehicles
}

Mohamed Maghenem, Antonio Loria, Elena Panteley

\section{To cite this version:}

Mohamed Maghenem, Antonio Loria, Elena Panteley. A Cascades Approach to Formation-Tracking Stabilization of Force-Controlled Autonomous Vehicles. IEEE Transactions on Automatic Control, 2018, 63 (8), pp.2662-2669. 10.1109/TAC.2017.2774003 . hal-01744645

\section{HAL Id: hal-01744645 \\ https://hal.science/hal-01744645}

Submitted on 5 Mar 2020

HAL is a multi-disciplinary open access archive for the deposit and dissemination of scientific research documents, whether they are published or not. The documents may come from teaching and research institutions in France or abroad, or from public or private research centers.
L'archive ouverte pluridisciplinaire $\mathbf{H A L}$, est destinée au dépôt et à la diffusion de documents scientifiques de niveau recherche, publiés ou non, émanant des établissements d'enseignement et de recherche français ou étrangers, des laboratoires publics ou privés. 


\title{
A cascades approach to formation-tracking stabilization of force-controlled autonomous vehicles
}

\author{
Mohamed Maghenem Antonio Loría Elena Panteley
}

\begin{abstract}
We present a cascades-based controller for the problem of formation-tracking control in a group of autonomous vehicles. We consider general models composed of a velocity kinematics equation and a generic force-balance equation. For each vehicle, a local controller ensures tracking of a reference generated by a leader vehicle. One or many vehicles may have access to the reference trajectories; each robot has one leader only, but may have several followers (spanning-tree topology). We establish uniform global asymptotic stability in closed loop for a wide class of controllers. Our analysis relies on the construction of an original strict Lyapunov function for the position tracking error dynamics and an inductive argument based on cascades-systems theory.
\end{abstract}

\section{INTRODUCTION}

For a group of autonomous vehicles, the formation-tracking control problem roughly consists in making them form a spatial configuration and move along a reference trajectory while keeping the pattern [1]. If the reference trajectory is generated by one of the agents we speak of the leader-follower formation-tracking control problem. This is a natural extension of the classical tracking control problem to the multi-agent framework.

For control design, autonomous vehicles are often modeled as unicycle systems, with two Cartesian coordinates for translation and one for orientation; this is the so-called kinematic model. Full models include an additional forces-balance equation, e.g., in Lagrangian form [2] or in Hamiltonian one [3]. Yet, even at the kinematic level, tracking control imposes certain difficulties when considering generic reference trajectories that stem from the nonholonomy of the robot [4].

The formation tracking control problem using a full dynamical model of the unicycle has been studied, e.g., in [5], [6], [7], [8], [9]. In [5], an output-feedback controller is used under the assumption that the leader translational velocity is separated from zero. Under the assumption that the robot is modeled as a point-mass (second-order integrators), a particular time-varying configuration is considered in [6]. In scenarios involving parametric uncertainty, a distributed formation controller has been proposed in [7] to solve the formation tracking problem, ensuring error convergence in a practical sense only. See also [8] where a general balanced directed communication graph is considered under the assumption that the rotational velocity of the leader is persistently exciting and a decentralized control law is proposed. In [9] skidding, slipping, and collision avoidance are considered under the assumption that the leader translational velocity is separated from zero.

In this note we address the problem of formation tracking control in a leader-follower topological configuration. That is, each robot follows one leader and communicates its coordinates to one or several followers that stand not necessarily physically close. The reference trajectory is generated by a virtual robot, which may be known by only one, or by several robots. In contrast to [10], the unicycle's full model is used. Our controllers are based on a recursive repetition of a time-varying nonlinear tracking controller for the kinematics

M. Maghenem is with Univ Paris-Saclay, A. Loria and E. Panteley are with the CNRS, France. L2S-CentraleSuplec, 91192 Gif-sur-Yvette, France. E-mail: loria@lss.supelec.fr. E. Panteley is also with ITMO University, Kronverkskiy av. 49, Saint Petersburg, 197101, Russia. This article is supported by Government of Russian Federation (grant 074-U01) model, based on [11]. In this case, we establish, via Lyapunov's direct method, that the origin is uniformly globally asymptotically stable. For the complete model, we show that any controller ensuring the stabilization at the force level with sufficiently fast rate of convergence, leads to uniform global asymptotic stability for the global dynamics.

Our main contributions lie in the construction of a strict Lyapunov function for the kinematics model and the proofs of uniform global asymptotic stability. Even though Lyapunov's direct method is also used, e.g., in [12], our main condition is that either the forward or the angular reference velocity is persistently exciting. This condition, which is the same as in [13] and [10], is weaker than what is usually considered in the literature. For instance, neither velocity is required to be separated from zero and one may equal to zero if the other is time-varying non-negative.

Our main results are stated in Section II; firstly for leader-follower tracking control and then, for formation tracking of large groups. The constructive stability proofs are provided in Section III, before concluding with some remarks in Section V.

\section{PROBLEM FORMULATION AND ITS SOLUTION}

\section{A. Tracking control}

Consider the force-controlled model of an autonomous vehicle:

$$
\begin{aligned}
& \left\{\begin{array}{l}
\dot{x}=v \cos \theta \\
\dot{y}=v \sin \theta \\
\dot{\theta}=\omega
\end{array}\right. \\
& \left\{\begin{array}{l}
\dot{v}=f_{1}(t, v, \omega, z)+u_{1} \\
\dot{\omega}=f_{2}(t, v, \omega, z)+u_{2}
\end{array}\right.
\end{aligned}
$$

where $v$ and $\omega$ denote the forward and angular velocities respectively,

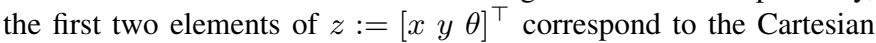
coordinates of a point on the robot with respect to a fixed reference frame, and $\theta$ denotes the robot's orientation with respect to the same frame. The functions $f_{1}$ and $f_{2}$ satisfy the Caratheodory conditions for existence of solutions and $u_{1}$ and $u_{2}$ denote the two torque control inputs.

Equations (1) correspond to the velocity kinematics model while (2) correspond to the force-balance equations. The control strategy in this paper consists in decoupling the stabilization task at both levels. For (1) we establish robustness results hence, our main statements are valid for any controller that guarantees the stabilization of (2) with "fast" convergence (for instance, but not only, local exponential).

The tracking-control problem consists in making the robot to follow a fictitious reference vehicle modeled by

$$
\begin{aligned}
& \dot{x}_{r}=v_{r} \cos \theta_{r} \\
& \dot{y}_{r}=v_{r} \sin \theta_{r} \\
& \dot{\theta}_{r}=\omega_{r},
\end{aligned}
$$

and which moves about with reference velocities $v_{r}(t)$ and $\omega_{r}(t)$. More precisely, it is desired to steer the differences between the Cartesian coordinates to some values $d_{x}, d_{y}$, and to zero the orientation angles and the velocities of the two robots, that is, the quantities

$$
p_{\theta}=\theta_{r}-\theta, \quad p_{x}=x_{r}-x-d_{x}, \quad p_{y}=y_{r}-y-d_{y} .
$$


The distances $d_{x}, d_{y}$ define the position of the robot with respect to the (virtual) leader. In general, these may be functions that depend on time and the state or may be assumed to be constant, depending on the desired path to be followed. In this paper, we consider these distances to be defined as piece-wise constant functions $-c f$. [14].

Then, as it is customary, we transform the error coordinates $\left[p_{\theta}, p_{x}, p_{y}\right]$ of the leader robot from the global coordinate frame to local coordinates fixed on the robot, that is, we define

$$
\left[\begin{array}{l}
e_{\theta} \\
e_{x} \\
e_{y}
\end{array}\right]:=\left[\begin{array}{ccc}
1 & 0 & 0 \\
0 & \cos \theta & \sin \theta \\
0 & -\sin \theta & \cos \theta
\end{array}\right]\left[\begin{array}{l}
p_{\theta} \\
p_{x} \\
p_{y}
\end{array}\right] .
$$

In these new coordinates, the error dynamics between the virtual reference vehicle and the follower becomes

$$
\begin{aligned}
& \dot{e}_{\theta}=\omega_{r}(t)-\omega \\
& \dot{e}_{x}=\omega e_{y}-v+v_{r}(t) \cos \left(e_{\theta}\right) \\
& \dot{e}_{y}=-\omega e_{x}+v_{r}(t) \sin \left(e_{\theta}\right),
\end{aligned}
$$

in addition to Eqs (2). Hence, the control problem reduces to steering the trajectories of (4) to zero via the inputs $u_{1}$ and $u_{2}$ in (2). A natural method consists in designing virtual control laws at the kinematics level, denoted $w^{*}$ and $v^{*}$, and control inputs $u_{1}$ and $u_{2}$, depending on $w^{*}, v^{*}, \dot{\omega}^{*}$, and $\dot{v}^{*}$, such that the origin $(e, \tilde{v}, \tilde{w})=(0,0,0)$ with

$$
\tilde{v}:=v-v^{*}, \quad \tilde{\omega}:=\omega-\omega^{*}, \quad e=\left[\begin{array}{lll}
e_{\theta} & e_{x} & e_{y}
\end{array}\right]^{\top},
$$

is uniformly globally asymptotically stable.

The stabilization problem for (4), that is, setting $\omega \equiv \omega^{*}$ and $v \equiv v^{*}$ and, hence, neglecting the dynamics (2), has been broadly studied in the literature. For instance, in [11] the authors proposed the controller

$$
\begin{aligned}
v^{*} & :=v_{r}(t) \cos \left(e_{\theta}\right)+k_{x} e_{x} \\
\omega^{*} & :=\omega_{r}(t)+k_{\theta} e_{\theta}+v_{r}(t) k_{y} e_{y} \phi\left(e_{\theta}\right)
\end{aligned}
$$

where $k_{x}, k_{y}$ and $k_{\theta}$ are positive control gains, $\phi$ is the so-called 'sinc' function defined by

$$
\phi\left(e_{\theta}\right):=\frac{\sin \left(e_{\theta}\right)}{e_{\theta}}
$$

and established (non-uniform) convergence of the tracking errors under the assumption that either $v_{r}$ or $\omega_{r}$ has a limit separated from zero. Then, for the same controller but under slightly relaxed conditions, uniform global asymptotic stability for the closed-loop system is established in [13]. In this paper, the same property is established and, in addition, for the first time a strict Lyapunov function is provided. As in [13], our standing assumption is that either the forward or the angular reference velocities are persistently exciting that is, that there exist positive numbers $\mu$ and $T$ such that

$$
\int_{t}^{t+T}\left[\omega_{r}(s)^{2}+v_{r}(s)^{2}\right] d s \geq \mu \quad \forall t \geq 0 .
$$

The design of the controller (6) is motivated by the resulting structure of the error dynamics for the tracking errors, which is reminiscent of nonlinear adaptive control systems. Indeed, by setting $\omega=\omega^{*}$ and $v=v^{*}$, we obtain

$$
\left[\begin{array}{c}
\dot{e}_{\theta} \\
\dot{e}_{x} \\
\dot{e}_{y}
\end{array}\right]=\underbrace{\left[\begin{array}{ccc}
-k_{\theta} & 0 & -v_{r}(t) k_{y} \phi\left(e_{\theta}\right) \\
0 & -k_{x} & \omega^{*}(t, e) \\
v_{r}(t) \phi\left(e_{\theta}\right) & -\omega^{*}(t, e) & 0
\end{array}\right]}_{A_{v_{r}}(t, e)}\left[\begin{array}{c}
e_{\theta} \\
e_{x} \\
e_{y}
\end{array}\right] \text {. }
$$

Then, we evaluate the total derivative of $V_{1}: \mathbb{R}^{3} \rightarrow \mathbb{R}_{\geq 0}$, defined as

$$
V_{1}(e)=\frac{1}{2}\left[e_{x}^{2}+e_{y}^{2}+\frac{1}{k_{y}} e_{\theta}^{2}\right]
$$

to obtain

$$
\dot{V}_{1}(e)=-k_{x} e_{x}^{2}-k_{\theta} e_{\theta}^{2} \leq 0
$$

and to conclude that the trivial solution for (9) is uniformly globally stable, that is, it is uniformly stable and all solutions are uniformly globally bounded.

Furthermore, after [15], it may be concluded that the origin of this system is uniformly globally asymptotically stable provided that the vector $\left[-v_{r}(t) k_{y} \phi\left(e_{\theta}\right) \quad \omega^{*}(t, e)\right]$, subject to $e_{\theta}=0$, is $\delta$ persistently exciting with respect to $e_{y}$. Roughly, this holds provided that this vector is persistently exciting for any $e_{y} \neq 0$; condition which, actually, reduces to (8). Thus, our first statement is the following.

Proposition 1 (Kinematic model): For the system (9) assume that (8) holds and there exist $\bar{\omega}_{r}, \overline{\dot{\omega}}_{r}, \bar{\nu}, \overline{\dot{\nu}}>0$ such that ${ }^{1}$

$$
\left|\omega_{r}\right|_{\infty} \leq \bar{\omega}_{r}, \quad\left|\dot{\omega}_{r}\right|_{\infty} \leq \overline{\dot{\omega}}_{r}, \quad\left|v_{r}\right|_{\infty} \leq \bar{v}_{r}, \quad\left|\dot{v}_{r}\right|_{\infty} \leq \overline{\dot{v}}_{r} .
$$

Then, the origin is uniformly globally asymptotically stable and locally exponentially stable, for any positive values of the control gains $k_{x}, k_{y}$, and $k_{\theta}$.

Beyond the statement itself, our (first) contribution lies in the original proof of Proposition 1 (see Section III) which is based on Lyapunov's direct method. Concretely, following the methods of [16], we show that there exists a positive definite radially unbounded function $V: \mathbb{R}_{\geq 0} \times \mathbb{R}^{3} \rightarrow \mathbb{R}_{\geq 0}$ defined as the functional

$$
V(t, e):=P_{[3]}\left(t, V_{1}\right) V_{1}(e)-\omega_{r}(t) e_{x} e_{y}+v_{r}(t) P_{[1]}\left(t, V_{1}\right) e_{\theta} e_{y}
$$

where, for any $k \in \mathbb{Z}$, we denote by $P_{[k]}: \mathbb{R}_{\geq 0} \times \mathbb{R}_{\geq 0} \rightarrow \mathbb{R}_{\geq 0}$ a smooth function such that $P_{[k]}\left(\cdot, V_{1}\right)$ is uniformly bounded and $P_{[k]}(t, \cdot)$ is a polynomial of degree $k$, with non-negative coefficients, designed such that the total derivative of $V$ along the trajectories of (9) satisfies

$$
\dot{V}(t, e) \leq-\frac{\mu}{T} V_{1}(e)-k_{x} e_{x}^{2}-k_{\theta} e_{\theta}^{2} .
$$

The value of having a strict Lyapunov function for (9) may not be overestimated. It serves to study the robustness of this error system when $\omega \not \equiv \omega^{*}$ and $v \not \equiv v^{*}$; in other words, the robustness of (9) visa-vis of the input $(\tilde{v}, \tilde{\omega})$. The dynamics of these variables are given by the equations (2) in closed loop with control inputs $u_{1}$ and $u_{2}$. In general, such closed-loop equations may be written as

$$
\begin{aligned}
\dot{\tilde{v}} & =f_{1 c l}(t, \tilde{v}, \tilde{\omega}, e) \\
\dot{\tilde{\omega}} & =f_{2 c l}(t, \tilde{v}, \tilde{\omega}, e)
\end{aligned}
$$

so that the overall error dynamics takes the convenient form

$$
\begin{aligned}
\dot{e} & =A_{v_{r}}(t, e) e+B(e) \eta, \\
\dot{\eta} & =F_{c l}(t, \tilde{v}, \tilde{\omega}, e), \quad F_{c l}:=\left[f_{1 c l} f_{2 c l}\right],
\end{aligned}
$$

where

$$
B(e):=\left[\begin{array}{cc}
0 & -1 \\
-1 & e_{y} \\
0 & -e_{x}
\end{array}\right], \quad \eta:=\left[\begin{array}{c}
\tilde{v} \\
\tilde{\omega}
\end{array}\right] .
$$

Now, for the purpose of analysis, we replace the state $e$ with the trajectories $e\left(t, \zeta\left(t_{\circ}\right)\right)$ in (16b) so the closed-loop equations may be regarded as a cascaded nonlinear time-varying system with state $\zeta:=\left[e^{\top} \eta^{\top}\right]^{\top}-c f$. [17]. More precisely, in place of (16b) we write

$$
\dot{\eta}=\tilde{F}_{c l}(t, \eta)
$$

where $\tilde{F}_{c l}(t, \eta)=F_{c l}(t, \tilde{v}, \tilde{\omega}, e(t))$. Thus, roughly speaking, provided that the origin for the nominal dynamics $\dot{e}=A_{v_{r}}(t, e)$ is asymptotically stable, and the input $\eta \rightarrow 0$ sufficiently fast and for

\footnotetext{
${ }^{1}$ For a measurable function $t \mapsto \psi$ we use $|\psi|_{\infty}:=\operatorname{ess} \sup _{t \geq 0}|\psi(t)|$.
} 
any $e$, a cascades argument applies to establish that $e \rightarrow 0$. This reasoning lies at the basis of our first statement:

Proposition 2: Consider the system (16) with initial conditions $\left(t_{\circ}, \zeta_{\circ}\right) \in \mathbb{R}_{>0} \times \mathbb{R}^{5}$. Assume that $k_{x}, k_{y}$, and $k_{\theta}$ are positive and that Inequalities (8) and (12) hold. In addition, assume that the solutions are complete and for the system (18) the origin, $\{\eta=0\}$, is uniformly globally asymptotically stable. Assume further that the trajectories $t \mapsto \eta$ are uniformly integrable, that is, there exists $\phi \in \mathcal{K}$ such that

$$
\int_{t_{\circ}}^{\infty}|\eta(\tau)| d \tau \leq \phi\left(\left|\zeta_{\circ}\right|\right) \quad \forall t \geq t_{\circ} \geq 0
$$

Then, the origin is uniformly globally asymptotically stable.

Proof. From Proposition 1, the origin $\{e=0\}$ is uniformly globally asymptotically stable for (9). By assumption the same property holds for (16b). Since, moreover, $B$ is linear in $e$, the result follows from [18, Theorem 2].

Remark 1: The following observations are in order:

- Since $\tilde{F}_{c l}$ in (18) depends on the trajectories $e(t)$ hence, uniformity is to be understood both with respect to the initial conditions and the trajectories $e(t)$.

- Technically, the function $\tilde{F}_{c l}$ is defined only on the interval of existence of $e(t)$, whence the assumption that the solutions exist on $\left[t_{\circ}, \infty\right)$. Nevertheless, this hypothesis may be dropped if we impose that $\eta \rightarrow 0$ uniformly in $e(t)$ only on the interval of existence. This is considered in our main result later on, Proposition 3.

- An example of feedbacks $u_{1}, u_{2}$ is given in Section II-C.

\section{B. Formation tracking control}

Let us consider now $n$ mobile robots that are required to advance in formation. It is assumed that the $i$ th robot follows a leader, indexed $i-1$, thereby forming a spanning-tree graph communication topology. That is, each robot has only one leader, but it may have several followers. As previously explained, the geometry of the formation may be defined via the relative distances between any pair of leaderfollower robots, $d_{x i}, d_{y i}$ and it is independent of the communications graph (two robots may communicate independently of their relative positions). Then, the relative position error dynamics is given by a set of equations similar to (4), that is,

$$
\begin{aligned}
\dot{e}_{\theta i} & =\omega_{i-1}(t)-\omega_{i} \\
\dot{e}_{x i} & =\omega_{i} e_{y i}-v_{i}+v_{i-1}(t) \cos \left(e_{\theta i}\right) \\
\dot{e}_{y i} & =-\omega_{i} e_{x i}+v_{i-1}(t) \sin \left(e_{\theta i}\right) .
\end{aligned}
$$

For $i=1$ we recover the tracking error dynamics for the case of one robot following a virtual leader that is, by definition, $v_{0}:=v_{r}$ and $\omega_{0}:=\omega_{r}$. Then, similarly to (6) we introduce the virtual controls

$$
\begin{aligned}
v_{i}^{*} & :=v_{i-1} \cos \left(e_{\theta i}\right)+k_{x i} e_{x i} \\
\omega_{i}^{*} & :=\omega_{i-1}+k_{\theta i} e_{\theta_{i}}+v_{i-1} k_{y i} e_{y i} \phi\left(e_{\theta i}\right)
\end{aligned}
$$

which serve as references for the actual controls $u_{1 i}$ and $u_{2 i}$ in

$$
\begin{aligned}
\dot{v}_{i} & =f_{1 i}\left(t, v_{i}, \omega_{i}, e_{i}\right)+u_{1 i} \\
\dot{\omega}_{i} & =f_{2 i}\left(t, v_{i}, \omega_{i}, e_{i}\right)+u_{2 i}, \quad i \leq n .
\end{aligned}
$$

Next, let us introduce the velocity errors

$$
\tilde{\omega}_{i}:=\omega_{i}-\omega_{i}^{*}, \quad \tilde{v}_{i}:=v_{i}-v_{i}^{*}
$$

and, for the purpose of analysis only, let us define $\Delta v_{j}:=v_{j}-v_{r}$ and $\Delta \omega_{j}:=\omega_{j}-\omega_{r}$ for all $j \leq n$ (by definition, $\Delta \omega_{0}=\Delta v_{0}=0$ ). Then, we replace $\omega_{i}$ with $\tilde{\omega}_{i}+\omega_{i}^{*}$ and, respectively, $v_{i}$ with $\tilde{v}_{i}+v_{i}^{*}$ in (20), and we use

$$
v_{i}^{*}=\left[\Delta v_{i-1}+v_{r}\right] \cos \left(e_{\theta i}\right)+k_{x i} e_{x i}
$$

$\omega_{i}^{*}=\Delta \omega_{i-1}+\omega_{r}+k_{\theta i} e_{\theta_{i}}+\left[\Delta v_{i-1}+v_{r}\right] k_{y i} e_{y i} \phi\left(e_{\theta i}\right)$.

It follows that, for each pair of nodes, the error system takes the form

$$
\dot{e}_{i}=A_{v_{r}}\left(t, e_{i}\right) e_{i}+G\left(t, e_{i}, \xi_{i}\right) e_{i}+B\left(e_{i}\right) \eta_{i}
$$

-cf. (16a), where

$$
\begin{aligned}
& e_{i}:=\left[\begin{array}{ll}
e_{\theta_{i}} & e_{x_{i}} e_{y_{i}}
\end{array}\right]^{\top}, \quad \eta_{i}:=\left[\begin{array}{ll}
\tilde{v}_{i} & \tilde{\omega}_{i}
\end{array}\right]^{\top} \\
& \xi_{i}:=\left[\Delta \omega_{i-1} \Delta v_{i-1}\right]^{\top} \\
& G:=\left[\begin{array}{ccc}
0 & 0 & -k_{y} g_{1} \\
0 & 0 & g_{2} \\
g_{1} & -g_{2} & 0
\end{array}\right] \\
& g_{1}:=\Delta v_{i-1} e_{y_{i}} \phi\left(e_{\theta_{i}}\right) \\
& g_{2}:=\Delta \omega_{i-1}+k_{y} \Delta v_{i-1} e_{y_{i}} \phi\left(e_{\theta_{i}}\right)
\end{aligned}
$$

and $B$ is defined in (17) —note that $G\left(t, e_{i}, 0\right) \equiv 0$. Thus, the overall closed-loop system has the convenient cascaded form (in reverse order):

$$
\begin{aligned}
\dot{e}_{n} & =A_{v_{r}}\left(t, e_{n}\right) e_{n}+G\left(t, e_{n}, \xi_{n}\right) e_{n}+B\left(e_{n}\right) \eta_{n} \\
& \vdots \\
\dot{e}_{2} & =A_{v_{r}}\left(t, e_{2}\right) e_{2}+G\left(t, e_{2}, \xi_{2}\right) e_{2}+B\left(e_{2}\right) \eta_{2} \\
\dot{e}_{1} & =A_{v_{r}}\left(t, e_{1}\right) e_{1}+B\left(e_{1}\right) \eta_{1}
\end{aligned}
$$

and these closed-loop equations are complemented by the equations that stem from applying the actual control inputs in (23), that is,

$$
\dot{\eta}_{i}=F_{i_{c l}}\left(t, \tilde{v}_{i}, \tilde{\omega}_{i}, e_{i}\right), \quad F_{i_{c l}}:=\left[\begin{array}{ll}
f_{i_{1 c l}} & f_{i_{2 c l}}
\end{array}\right]
$$

for all $i \leq n$.

To underline the good structural properties of the system (27)(28) and to explain the rationale of our main result, let us argue as follows (precise proofs are given in Section III). By assumption, the control inputs $u_{1 i}$ and $u_{2 i}$ are such that $\eta_{i} \rightarrow 0$, independently of the behavior of $e_{i}$. Furthermore, we see from Equation (27c) that, as $\eta_{1} \rightarrow 0$, we recover the system (9). Hence, if $\eta_{1}$ converges fast enough (according to (19)), using Proposition 2 we may conclude that $\eta_{1} \rightarrow 0$ implies that $e_{1} \rightarrow 0$. With this in mind, let us observe (27b). We have $\xi_{2}:=\left[\begin{array}{ll}\Delta \omega_{1} & \Delta v_{1}\end{array}\right]^{\top}$ where $\Delta \omega_{1}=\omega_{1}-\omega_{r}$ and $\Delta v_{1}=v_{1}-v_{r}$. On the other hand, by virtue of the control design, $e_{1}=0$ implies that $\omega_{1}^{*}=\omega_{r}$ and $v_{1}^{*}=v_{r}$, in which case we have $\Delta \omega_{1}=\tilde{\omega}_{1}$ and $\Delta v_{1}=\tilde{v}_{1}$. It follows that $e_{1} \rightarrow 0$ and $\eta_{1} \rightarrow 0$ imply that $\xi_{2} \rightarrow 0$. In addition, as $\eta_{2} \rightarrow 0$ (by the action of the controller at the force level), the terms $G\left(t, e_{2}, \xi_{2}\right) e_{2}+B\left(e_{2}\right) \eta_{2}$ in (27b) vanish and (27b) becomes $\dot{e}_{2}=A_{v_{r}}\left(t, e_{2}\right) e_{2}$. By Proposition 1 we conclude that $e_{2}$ also tends to zero. Carrying on by induction, we conclude that $e \rightarrow 0$.

Although intuitive, the previous arguments implicitly rely on the robustness of $\dot{e}_{i}=A_{v_{r}}\left(t, e_{i}\right)$ (i.e., of the system (9)) with respect to the inputs $\eta_{i}$ and $\xi_{i}$. More precisely, on the condition that the solutions exist on $\left[t_{\circ}, \infty\right)$ and, moreover, that they remain uniformly bounded during the transient. In our main result, which is presented next, we relax these (technical) assumptions. The proof is presented in Section III. It may also be showed that each subsystem in (27) is integral-input-to-state stable [19].

Proposition 3: For each $i \leq n$, let $\zeta_{i}:=\left[\begin{array}{ll}e_{i}^{\top} & \eta_{i}^{\top}\end{array}\right]^{\top}$ and consider the system (20), (23). Let conditions (8) and (12) hold and assume that control control inputs $u_{1 i}$ and $u_{2 i}$ are given, as functions of the states of the $i$ th robot and its leader $i-1$ st, such that:

\footnotetext{
${ }^{2}$ If necessary, we consider the shortest maximal interval of existence among all the trajectories $e_{i}(t)$, with $i \leq n$
} 
[A1] for each $i$, there exists a function $\beta_{i} \in \mathcal{K} \mathcal{L}$ such that, on the maximal interval of existence ${ }^{1}$ of $t \mapsto e_{i}$,

$$
\left|\eta_{i}\left(t, t_{\circ}, \eta_{1 \circ}, e_{i \circ}\right)\right| \leq \beta\left(\left|\zeta_{i \circ}\right|, t-t_{\circ}\right)
$$

and (19) holds for some $\phi_{i} \in \mathcal{K}$

Then, $\{\zeta=0\}$, where $\zeta:=\left[\zeta_{1}^{\top} \cdots \zeta_{n}^{\top}\right]^{\top}$, is uniformly globally asymptotically stable.

Remark 2: In Proposition 3 the control inputs $u_{1 i}$ and $u_{2 i}$, which typically depend on $v_{i}^{*}, \omega_{i}^{*}, \dot{v}_{i}^{*}$, and $\dot{\omega}_{i}^{*}$, are not explicitly defined on purpose; the statement is general. For instance, the most obvious choice for $u_{1 i}$ and $u_{2 i}$ corresponds to the linearizing feedbacks $u_{1 i}:=\dot{v}_{i}^{*}-f_{1 i}\left(t, v_{i}, \omega_{i}, e_{i}\right)-k_{1} \tilde{v}_{i}$ and $u_{2 i}=\dot{\omega}_{i}^{*}-f_{2 i}\left(t, v_{i}, \omega_{i}, e_{i}\right)-$ $k_{2} \tilde{\omega}_{i}$, but, in general, the design of $u_{1 i}$ and $u_{2 i}$ depends on the problem setting (parametric uncertainty, partial-state measurements, ...) and is beyond the scope of this technical note.

The virtual controls $v_{i}^{*}$ and $\omega_{i}^{*}$ are defined as in Eqs. (21) and (22), as functions of the leader velocities and the leader-follower relative position errors, while the accelerations $\dot{v}_{i}^{*}$ and $\dot{\omega}_{i}^{*}$ may be computed by differentiating (21) and (22) and using the leader's velocities and accelerations. Hence, the distributed controllers for each follower depend only on its own measurements and those of the leader; the equivalent expressions (24) and (25), which exhibit a recursive dependence of the leader's leader variables, are provided for the purpose of analysis only.

Remark 3: Assumption A1 means that $\eta_{i}(t)$ converge uniformly to zero while the trajectories $e_{i}(t)$ exist. In particular, if the system is forward complete A1 imposes uniform global asymptotic stability of (28). Even though this may be a strong hypothesis in a general context of nonlinear systems -see [17], it may be easily met in the case of formation tracking control, as we show below.

\section{Example}

As explained in the Introduction, the equations (2) correspond to a generic dynamics model which may be expressed, e.g., in Hamiltonian coordinates — see [3], or in Lagrangian ones — see [2]. The statement of Proposition 3 is general in the sense that it applies to any stabilizing controller for the equations (2). For illustration, let us consider the particular model of a wheeled mobile robot in Lagrangian coordinates $-c f$. [20],

$$
\begin{aligned}
& \dot{z}=J(z) \nu \\
& M \dot{\nu}+C(\dot{z}) \nu+D \nu=\tau
\end{aligned}
$$

where $z=[x, y, \theta]$ corresponds to the Cartesian position coordinates and the orientation, $\tau$ is the torque control input, the variable $\nu:=$ $\left[\begin{array}{ll}\nu_{1} & \nu_{2}\end{array}\right]$ denotes the angular velocities of the two wheels, $M$ is an inertia matrix (hence positive definite, symmetric), $C$ is the matrix of Coriolis forces (which is skew-symmetric), $D \nu$ with $D=D^{\top} \geq 0$ represents damping forces, and

$$
J(z)=\frac{r}{2}\left[\begin{array}{cc}
\cos \theta & -\sin \theta \\
\sin \theta & \cos \theta \\
1 / b & -1 / b
\end{array}\right]
$$

where $r$ and $b$ are positive constant parameters of the system. The relation between the wheels' velocities, $\nu$, and the robot's velocities in the fixed frame, $\dot{z}$, is given by

$$
\left[\begin{array}{l}
v \\
\omega
\end{array}\right]=\frac{r}{2 b}\left[\begin{array}{cc}
b & b \\
1 & -1
\end{array}\right]\left[\begin{array}{l}
\nu_{1} \\
\nu_{2}
\end{array}\right] \Leftrightarrow\left[\begin{array}{l}
\nu_{1} \\
\nu_{2}
\end{array}\right]=\frac{1}{r}\left[\begin{array}{cc}
1 & b \\
1 & -b
\end{array}\right]\left[\begin{array}{c}
v \\
\omega
\end{array}\right]
$$

which may be used in (30) to obtain the model (1), (2) with

$$
\left[\begin{array}{l}
u_{1} \\
u_{2}
\end{array}\right]=\frac{r}{2 b}\left[\begin{array}{cc}
b & b \\
1 & -1
\end{array}\right] M^{-1} \tau
$$

— see [20] for more details on this coordinate transformation.
Then, using (31), for any given virtual control inputs $v^{*}$ and $\omega^{*}$, we can compute $\nu^{*}:=\left[\begin{array}{ll}\nu_{1}^{*} & \nu_{2}^{*}\end{array}\right]^{\top}$ and define the torque control input

$$
\tau=M \dot{\nu}^{*}+C(J(z) \nu) \nu^{*}+D \nu^{*}-k_{d} \tilde{\nu}, \quad k_{d}>0
$$

where $\tilde{\nu}:=\nu-\nu^{*}$. We see that the force error equations yields

$$
M \dot{\tilde{\nu}}+\left[C(\dot{z}(t))+D+k_{d} I\right] \tilde{\nu}=0
$$

in which we have replaced $\dot{z}$ with the trajectories $\dot{z}(t)$ to regard this system as (linear) time-varying, with state $\tilde{\nu}$. Now, due to the skewsymmetry of $C(\cdot)$ the total derivative of

$$
V(\tilde{\nu})=\frac{1}{2} \tilde{\nu}^{\top} M \tilde{\nu}
$$

along the trajectories of (33) yields

$$
\dot{V}(\tilde{\nu}) \leq-k_{d}|\tilde{\nu}|^{2} .
$$

Although this inequality holds independently of $\dot{z}(t)$, Eq. (33) is valid only on the interval of existence of $\dot{z}(t)$, denoted $\left[t_{\circ}, t^{\max }\right)$, $t^{\max } \leq \infty$. Hence, so does (34) and, consequently,

$$
|\tilde{\nu}(t)| \leq \kappa\left|\tilde{\nu}\left(t_{\circ}\right)\right| e^{-\lambda\left(t-t_{\circ}\right)} \quad \forall t \in\left[t_{\circ}, t^{\max }\right)
$$

for some $\kappa$ and $\lambda>0$. From (31) it is clear that a similar bound holds for $\eta(t)=[\tilde{v}(t) \tilde{\omega}(t)]$. In other words, the velocity errors tend exponentially to zero uniformly in the initial conditions and in the position error trajectories, so condition A1 of Proposition 3 holds.

Remark 4: For the controller (32) it is assumed that all the constant lumped parameters are known. However, an adaptive controller for Lagrangian systems, such as the well-known Slotine \& Li controller [21] may be used. Moreover, uniform global asymptotic stability may be ensured (that is, including the convergence of the parameter estimation errors) provided that the reference trajectories $v^{*}$ are persistently exciting — see [22].

\section{STABILITY ANALYSIS}

\section{A. Proof of Proposition 1}

The proof follows via Lyapunov's direct method; it relies on the construction of a Lyapunov function of polynomial type, and it is greatly inspired by the methods in [16].

Firstly, for any locally integrable function $\varphi: \mathbb{R}_{\geq 0} \rightarrow \mathbb{R}_{\geq 0}$, such that $\sup _{t \geq 0}|\varphi(t)| \leq \bar{\varphi}$, let us introduce

$$
Q_{\varphi}(t):=1+2 \bar{\varphi} T-\frac{2}{T} \int_{t}^{t+T} \int_{t}^{m} \varphi(s) d s d m .
$$

Note that this function satisfies:

$$
\begin{gathered}
\dot{Q}_{\varphi}(t)=-\frac{2}{T} \int_{t}^{t+T} \varphi(s) d s+2 \varphi(t), \\
1 \leq Q_{\varphi}(t)<\bar{Q}_{\varphi}:=1+2 \bar{\varphi} T .
\end{gathered}
$$

In the sequel, we use this function with $\varphi=v_{r}^{2}+\omega_{r}^{2}$.

We also introduce several polynomial functions with positive coefficients, denoted by $\rho_{i}: \mathbb{R}_{\geq 0} \rightarrow \mathbb{R}_{\geq 0}$. These shall be defined as needed in a manner that the derivative of

$$
\begin{aligned}
V_{2}(t, e):= & \rho_{1}\left(V_{1}\right) V_{1}+\left[Q_{v_{r}^{2}}(t)+Q_{\omega_{r}^{2}}(t)\right] V_{1}-\omega_{r}(t) e_{x} e_{y} \\
& +v_{r} \rho_{2}\left(V_{1}\right) e_{\theta} e_{y}+\rho_{3}\left(V_{1}\right) V_{1},
\end{aligned}
$$

with $V_{1}$ defined in (10), be negative definite. In addition, note that

$$
V_{2}(t, e) \geq \frac{1}{2}\left[\begin{array}{l}
e_{\theta} \\
e_{x} \\
e_{y}
\end{array}\right]^{\top}\left[\begin{array}{ccc}
\rho_{3}\left(V_{1}\right) & v_{r} \rho_{2}\left(V_{1}\right) & 0 \\
v_{r} \rho_{2}\left(V_{1}\right) & \rho_{3}\left(V_{1}\right) & -\omega_{r} \\
0 & -\omega_{r} & \rho_{3}\left(V_{1}\right)
\end{array}\right]\left[\begin{array}{l}
e_{\theta} \\
e_{x} \\
e_{y}
\end{array}\right]
$$


so $V_{2}$ is positive definite and radially unbounded if the matrix in this inequality is positive semidefinite. The latter holds if $\rho_{3}$ satisfies

$$
\rho_{3}\left(V_{1}\right) \geq \frac{\sqrt{\bar{v}_{r}^{2} \rho_{2}\left(V_{1}\right)^{2}+\bar{\omega}_{r}^{2}}}{\sqrt{2}} .
$$

Finally, we introduce $V_{3}(t, e)=V_{2}(t, e)+V_{1} \rho_{4}\left(V_{1}\right)$, which is also positive definite. We shall show that for an appropriate choice of the polynomials $\rho_{i}$, the total derivative of $V_{3}$ along the trajectories of (9) yields

$$
\dot{V}_{3}(t, e) \leq-\frac{\mu}{T} V_{1}(e)-k_{x} e_{x}^{2}-k_{\theta} e_{\theta}^{2}, \quad \forall(t, e) \in \mathbb{R}_{\geq 0} \times \mathbb{R}^{3} .
$$

To that end, we start by rewriting (9) in the output-injection form

$$
\begin{aligned}
\dot{e} & =A_{v_{r}}^{\circ}(t, e) e+v_{r}\left[\phi\left(e_{\theta}\right)-1\right] B^{\circ}\left(e_{y}\right) e \\
A_{v_{r}}^{\circ}(t, e) & :=\left[\begin{array}{ccc}
-k_{\theta} & 0 & -v_{r} k_{y} \\
0 & -k_{x} & \varpi_{v_{r}}^{\circ} \\
v_{r} & -\varpi_{v_{r}}^{\circ} & 0
\end{array}\right] \\
B^{\circ}(e) & :=\left[\begin{array}{ccc}
0 & 0 & -k_{y} \\
0 & 0 & k_{y} e_{y} \\
1 & -k_{y} e_{y} & 0
\end{array}\right] \\
\varpi_{v_{r}}^{\circ}(t, e) & =\omega_{r}(t)+k_{\theta} e_{\theta}+v_{r} k_{y} e_{y} .
\end{aligned}
$$

This partition, which facilitates the analysis, is motivated by the fact that $v_{r}\left[\phi\left(e_{\theta}\right)-1\right] B^{\circ}\left(e_{y}\right) e=0$ if $e_{\theta}=0$.

Now we show that the total derivative of $V_{2}$ along the trajectories of $\dot{e}=A_{v_{r}}^{\circ}(t, e) e$ is negative definite. Firstly, since $\rho_{1}$ is a polynomial that maps $\mathbb{R}_{\geq 0} \rightarrow \mathbb{R}_{\geq 0}$ and $V_{1}$ satisfies (11),

$$
\frac{d}{d t}\left\{\rho_{1}\left(V_{1}\right) V_{1}\right\} \leq-\rho_{1}\left(V_{1}\right)\left[k_{x} e_{x}^{2}+k_{\theta} e_{\theta}^{2}\right] .
$$

Next, we use (36), as well as $|e| \geq\left|e_{y}\right|$ and $Q_{\varphi}>0$, to obtain

$$
\begin{aligned}
\frac{d}{d t}\left\{\left[Q_{v_{r}^{2}}+Q_{\omega_{r}^{2}}\right] V_{1}\right\} \leq- & \frac{2}{T}\left[\int_{t}^{t+T}\left[\omega_{r}(s)^{2}+v_{r}(s)^{2}\right] d s\right] V_{1} \\
& +\left[\omega_{r}^{2}+v_{r}^{2}\right]\left[e_{x}^{2}+\frac{1}{k_{y}} e_{\theta}^{2}+e_{y}^{2}\right]
\end{aligned}
$$

Then, using (40) and (42), we obtain

$$
\begin{gathered}
-\frac{d}{d t}\left\{\omega_{r} e_{x} e_{y}\right\}=-\dot{\omega}_{r} e_{x} e_{y}-\omega_{r}\left[-k_{x} e_{x} e_{y}+\omega_{r} e_{y}^{2}+k_{\theta} e_{\theta} e_{y}^{2}\right. \\
\left.+k_{y} v_{r} e_{y}^{3}-\omega_{r} e_{x}^{2}-k_{\theta} e_{\theta} e_{x}^{2}-k_{y} v_{r} e_{y} e_{x}^{2}+v_{r} e_{\theta} e_{x}\right] .
\end{gathered}
$$

Now, for the cross-terms we use the inequalities $2 e_{x} e_{y} \leq \epsilon e_{x}^{2}+$ $(1 / \epsilon) e_{y}^{2}$ and $2 e_{\theta} e_{y}^{2} \leq \epsilon V_{1} e_{\theta}^{2}+(1 / \epsilon) e_{y}^{2}$, which hold for any $\epsilon>0$, and we regroup some terms to obtain (see [19] for details)

$$
\begin{aligned}
& -\frac{d}{d t}\left\{\omega_{r} e_{x} e_{y}\right\} \leq \frac{\epsilon}{2} v_{r}^{2} V_{1} e_{y}^{2}+\rho_{5}\left(V_{1}\right) e_{x}^{2}+\rho_{6}\left(V_{1}\right) e_{\theta}^{2} \\
& \quad+\frac{1}{2 \epsilon}\left[\bar{\omega}_{r}^{2} k_{y}^{2}+\left(k_{x}+k_{\theta}\right) \bar{\omega}_{r}+\bar{\omega}_{r} k_{y} \bar{v}_{r}+\bar{\omega}_{r}\right] e_{y}^{2}-\omega_{r}^{2} e_{y}^{2}
\end{aligned}
$$

where $\rho_{5}$ and $\rho_{6}$ are first-order polynomials of $V_{1}$ defined as

$$
\begin{aligned}
\rho_{5}\left(V_{1}\right) & =\frac{\bar{\omega}_{r}}{2}\left[\left(\epsilon k_{y} \bar{v}_{r}+2 k_{\theta}\right) V_{1}+\left(k_{x}+\frac{\overline{\dot{\omega}}_{r}}{\bar{\omega}_{r}}\right) \epsilon+2 \bar{\omega}_{r}+\bar{v}_{r}\right] \\
\rho_{6}\left(V_{1}\right) & =\bar{\omega}_{r}\left[k_{\theta}\left(\epsilon V_{1}+1\right)+\frac{\bar{v}_{r}}{2}\right] .
\end{aligned}
$$

Next, we compute

$$
\begin{gathered}
\frac{d}{d t}\left\{v_{r} \rho_{2}\left(V_{1}\right) e_{\theta} e_{y}\right\}=-\rho_{2}\left(V_{1}\right) v_{r}^{2} e_{y}^{2}-v_{r} \rho_{2}\left(V_{1}\right)\left[k_{\theta} e_{\theta} e_{y}+\omega_{r} e_{x} e_{\theta}\right. \\
\left.+k_{\theta} e_{\theta}^{2} e_{x}+k_{y} v_{r} e_{y} e_{x} e_{\theta}+v_{r} e_{\theta}^{2}\right]+\rho_{2}\left(V_{1}\right) \dot{v}_{r} e_{\theta} e_{y} \\
-v_{r} \frac{\partial \rho_{2}}{\partial V_{1}} e_{\theta} e_{y}\left[k_{x} e_{x}^{2}+k_{\theta} e_{\theta}^{2}\right] .
\end{gathered}
$$

Hence, using again the triangle inequality to bound the cross-terms and regrouping them, we obtain

$$
\frac{d}{d t}\left\{v_{r} \rho_{2} e_{\theta} e_{y}\right\} \leq-k_{y} v_{r}^{2} \rho_{2}\left(V_{1}\right) e_{y}^{2}+\rho_{7}\left(V_{1}\right) e_{x}^{2}
$$

$$
+\rho_{8}\left(V_{1}\right) e_{\theta}^{2}+\frac{k_{\theta} \bar{v}_{r}+\overline{\dot{v}}_{r}}{2 \epsilon} e_{y}^{2},
$$

where $\rho_{7}$ and $\rho_{8}$ are second-order polynomials of $V_{1}$ satisfying

$$
\begin{aligned}
\rho_{7}\left(V_{1}\right) \geq & \rho_{2} \bar{v}_{r}\left[\frac{\bar{\omega}_{r}}{2}+\left(k_{\theta}+k_{y} \bar{v}_{r}\right) V_{1}\right] \\
& \quad+\max \left\{k_{y}, 1\right\} k_{x} \bar{v}_{r} V_{1}\left|\frac{\partial \rho_{2}}{\partial V_{1}}\right| \\
\rho_{8}\left(V_{1}\right) \geq & \frac{\bar{v}_{r} \rho_{2}\left(V_{1}\right)}{2}\left[\bar{\omega}_{r}+k_{\theta}\left(\epsilon \rho_{2}\left(V_{1}\right)+1\right)+\left(k_{y}+2\right) \bar{v}_{r}\right] \\
& \overline{\dot{v}}_{r} \frac{\epsilon}{2} \rho_{2}\left(V_{1}\right)^{2}+\bar{v}_{r}\left|\frac{\partial \rho_{2}}{\partial V_{1}}\right| \max \left\{k_{y}, 1\right\} k_{\theta} V_{1} .
\end{aligned}
$$

Now we put all the previous bounds together. Using (8) in (44), we obtain, in view of (46) and (48),

$$
\begin{aligned}
\frac{\partial V_{2}}{\partial t} & +\frac{\partial V_{2}}{\partial e} A_{v_{r}}^{\circ}(t, e) e \leq-\frac{2 \mu}{T} V_{1}(e)-\left[k_{y} \rho_{2}\left(V_{1}\right)-1-\frac{\epsilon}{2} V_{1}\right] v_{r}^{2} e_{y}^{2} \\
& +\frac{1}{2 \epsilon}\left[\bar{\omega}_{r}\left[\bar{\omega}_{r} k_{y}^{2}+k_{x}+k_{\theta}+k_{y} \bar{v}_{r}\right]+\overline{\dot{\omega}}_{r}+k_{\theta} \bar{v}_{r}+\overline{\dot{v}}_{r}\right] e_{y}^{2} \\
& -e_{x}^{2}\left[k_{x} \rho_{1}-\rho_{7}-\rho_{5}-v_{r}^{2}-\omega_{r}^{2}\right] \\
& -e_{\theta}^{2}\left[k_{\theta} \rho_{1}-\rho_{8}-\rho_{6}-\frac{1}{k_{y}}\left(v_{r}^{2}+\omega_{r}^{2}\right)\right] .
\end{aligned}
$$

Hence, defining

$$
\epsilon:=\frac{T}{\mu}\left[\bar{\omega}_{r}\left[\bar{\omega}_{r} k_{y}^{2}+k_{x}+k_{\theta}+k_{y} \bar{v}_{r}\right]+\overline{\dot{\omega}}_{r}+k_{\theta} \bar{v}_{r}+\overline{\dot{v}}_{r}\right]
$$

$\rho_{1}\left(V_{1}\right):=1+\frac{1}{\min \left\{k_{x}, k_{\theta}\right\}}\left[\rho_{5}+\rho_{6}+\rho_{7}+\rho_{8}\right.$

$$
\left.+\left[1+\frac{1}{k_{y}}\right]\left[\omega_{r}^{2}+v_{r}^{2}\right]\right],
$$

and

$$
\rho_{2}\left(V_{1}\right):=\frac{1}{k_{y}}\left[1+\frac{\epsilon}{2} V_{1}\right],
$$

we obtain

$$
\frac{\partial V_{2}}{\partial t}+\frac{\partial V_{2}}{\partial e} A_{v_{r}}^{\circ}(t, e) e \leq-\frac{\mu}{T} V_{1}(e)-k_{x} e_{x}^{2}-k_{\theta} e_{\theta}^{2} .
$$

That is, $V_{2}$ is a strict Lyapunov function for the nominal dynamics $\dot{e}=A_{v_{r}}^{\circ}(t, e) e$.

Next, we evaluate the total derivative of $V_{3}$ along the trajectories of (39) (i.e., including the output injection term). From (50) we obtain

$$
\begin{aligned}
& \dot{V}_{3}(t, e) \leq \frac{\partial V_{2}}{\partial t}+\frac{\partial V_{2}}{\partial e} A_{v_{r}}^{\circ}(t, e) e+W(t, e) \\
& W(t, e):=-k_{\theta} \rho_{4}\left(V_{1}\right) e_{\theta}^{2}+v_{r}\left[\phi\left(e_{\theta}\right)-1\right] \frac{\partial V_{2}}{\partial e} B^{\circ}\left(e_{y}\right) e
\end{aligned}
$$

for which we used (11), as well as the positivity of $\rho_{4}\left(V_{1}\right)$ and of $\partial \rho_{4} / \partial V_{1}$, to obtain

$$
\begin{aligned}
\frac{d}{d t}\left\{V_{1} \rho_{4}\left(V_{1}\right)\right\} & =\dot{V}_{1} \rho_{4}\left(V_{1}\right)+V_{1} \frac{\partial \rho_{4}}{\partial V_{1}} \dot{V}_{1} \\
& \leq-k_{\theta} \rho_{4}\left(V_{1}\right) e_{\theta}^{2} .
\end{aligned}
$$

We show that $W(t, e)$, defined in (52), is non-positive. To that end, note that

$$
\left[\phi\left(e_{\theta}\right)-1\right] \leq e_{\theta}^{2}
$$

and, in view of the structure of $B^{\circ}$, we have

$$
\frac{\partial V_{1}}{\partial e} B^{\circ}(e) e=0
$$

hence,

$$
\frac{\partial V_{2}}{\partial e}=v_{r} \rho_{2}\left(V_{1}\right)\left[\begin{array}{lll}
e_{y} & 0 & e_{\theta}
\end{array}\right]-\omega_{r}\left[\begin{array}{lll}
0 & e_{y} & e_{x}
\end{array}\right]
$$

and, moreover,

$$
\left|\left[\begin{array}{lll}
e_{y} & 0 & e_{\theta}
\end{array}\right] B^{\circ}(e) e\right| \leq\left|e_{\theta}^{2}-\frac{k_{y}}{2} e_{y}^{2}+\frac{k_{y}}{2} e_{x}^{2} e_{\theta}^{2}\right|
$$




$$
\begin{aligned}
& \leq 2 k_{y} V_{1}+2 k_{y}^{2} V_{1}^{2} \\
&\left|\left[\begin{array}{lll}
0 & e_{y} & e_{x}
\end{array}\right] B^{\circ}(e) e\right| \leq 2 k_{y} V_{1}^{2}+\max \left\{k_{y}, 1\right\} V_{1} .
\end{aligned}
$$

Thus, $W(t, e) \leq 0$ if

$$
\rho_{4}\left(V_{1}\right) \geq \frac{2 \bar{v}_{r} \max \left\{k_{y}, 1\right\}}{k_{\theta}}\left[\left[k_{y} \rho_{2} \bar{v}_{r}+\bar{\omega}_{r}\right] V_{1}^{2}+\left[\rho_{2} \bar{v}_{r}+\bar{\omega}_{r}\right] V_{1}\right]
$$

and (38) follows from (50) and (51).

Remark 5: From the definition of $V_{3}$ and (38) we see that for each compact $M \subset \mathbb{R}^{3}$, there exist constants $\alpha_{i}>0$ such that

$$
\begin{gathered}
\alpha_{1}|e|^{2} \leq V_{3}(t, e) \leq \alpha_{2}|e|^{2} \\
\dot{V}_{3}(t, e) \leq-\alpha_{3}|e|^{2}
\end{gathered}
$$

hence, the origin is exponentially stable on $M$. The computation of these constants in function of the control gains, of $\mu$, and $T$, may serve, as a guideline for gain tuning.

\section{B. Proof of Proposition 3}

The proof follows along the arguments developed below (28). For $i=1$ the closed-loop dynamics, composed of (27c) and

$$
\dot{\eta}_{1}=F_{1_{c l}}\left(t, \tilde{v}_{1}, \tilde{\omega}_{1}, e_{1}(t)\right)
$$

is defined on the interval of existence of $e_{1}(t)$, denoted $\left[t_{\circ}, t_{\max }\right)$, and has a cascaded form. By assumption, $\eta_{1}$ satisfies the bound (29) for all $t \in\left[t_{\circ}, t_{\max }\right)$ hence, on this interval,

$$
\begin{aligned}
\dot{V}_{1}\left(e_{1}(t)\right) & \leq \frac{\partial V_{1}}{\partial e_{1}}\left(e_{1}(t)\right) B\left(e_{1}(t)\right) \eta_{1}(t) \\
& \leq c V_{1}\left(e_{1}(t)\right) \sup _{t \in\left[t_{\circ}, t_{\max }\right)}\left|\eta_{1}(t)\right| \\
& \leq c^{\prime} V_{1}\left(e_{1}(t)\right)
\end{aligned}
$$

where $c$ and $c^{\prime}$ are positive constants of innocuous values, independent of the initial time. Integrating on both sides of the latter from $t_{\circ}$ to $t_{\max }$ we see that, by continuity of the solutions with respect to the initial conditions, this interval of integration may be stretched to infinity. By the definition of $V_{1}\left(e_{1}\right)$ we obtain that $e_{1}(t)$ exists on $\left[t_{\circ}, \infty\right)$. Moreover, since by definition $\Delta v_{0}=\Delta \omega_{0}=0$, we conclude from (24) and (25), that $v_{1}^{*}$ and $\omega_{1}^{*}$ exist along trajectories on $\left[t_{\circ}, \infty\right)$. It follows that the same property holds for $v_{1}(t)$ and $\omega_{1}(t)$ and, consequently, for $\xi_{2}(t)$-recall that

$$
\xi_{2}:=\left[\begin{array}{c}
v_{1}-v_{r} \\
\omega_{1}-\omega_{r}
\end{array}\right]
$$

From forward completeness and condition A1 it follows, in turn, that $\eta_{1}=0$ is uniformly globally asymptotically stable for (56).

Now we can apply a cascades argument for the system (27c), (56). Since $B$ in (27c) is linear in $e_{1}$ and the origin of $\dot{e}_{1}=A_{v_{r}}\left(t, e_{1}\right)$ is uniformly globally asymptotically stable, the same property holds for the origin $\left(e_{1}, \eta_{1}\right)=(0,0)$ - see [18, Theorem 2]. This means that there exists a class $\mathcal{K} \mathcal{L}$ function $\beta$ such that

$$
\left|\zeta_{1}\left(t, t_{\circ}, \zeta_{1 \circ}\right)\right| \leq \beta\left(\left|\zeta_{1 \circ}\right|, t-t_{\circ}\right) \quad \forall t \geq t_{\circ}
$$

where we recall that $\zeta_{i}=\left[e_{i}^{\top} \eta_{i}^{\top}\right]$ for all $i \leq n$. In particular, $e_{1}(t)$, $\eta_{1}(t)$ and, consequently, $\xi_{2}(t)$, are uniformly globally bounded. To see this more clearly, we recall that, by definition, $\xi_{2}$ is a continuous function of the state $\zeta_{1}$ and time and equals to zero if $\zeta_{1}=0$. Indeed, $\xi_{2}=\psi_{1}\left(t, \zeta_{1}\right)$ where

$$
\begin{aligned}
\psi_{1}\left(t, \zeta_{1}\right) & =\left[\begin{array}{c}
\tilde{v}_{1}+v_{1}^{*}-v_{r} \\
\tilde{\omega}_{1}+\omega_{1}^{*}-\omega_{r}
\end{array}\right] \\
& =\left[\begin{array}{l}
\tilde{v}_{1}+v_{r}(t)\left[\cos \left(e_{\theta 1}\right)-1\right]+k_{x 1} e_{x 1} \\
\tilde{\omega}_{1}+k_{\theta 1} e_{\theta_{1}}+v_{r}(t) k_{y 1} e_{y 1} \phi\left(e_{\theta 1}\right)
\end{array}\right] .
\end{aligned}
$$

Next, let $i=2$ and consider the closed-loop equations:

$$
\begin{aligned}
& \dot{e}_{2}=A_{v_{r}}\left(t, e_{2}\right) e_{2}+G\left(t, e_{2}, \psi_{1}\left(t, \zeta_{1}\right)\right) e_{2}+B\left(e_{2}\right) \eta_{2} \\
& \dot{\zeta}_{1}=F_{\zeta_{1}}\left(t, \zeta_{1}\right) \\
& \dot{\eta}_{2}=F_{2_{c l}}\left(t, \tilde{v}_{2}, \tilde{\omega}_{2}, e_{2}(t)\right)
\end{aligned}
$$

Note that we replaced $e_{2}$ with $e_{2}(t)$ in (28) to obtain the "decoupled" dynamics equation (62c). Then, $\eta_{2}$ is regarded as a perturbation to the system

$$
\begin{aligned}
\dot{e}_{2} & =A_{v_{r}}\left(t, e_{2}\right) e_{2}+G\left(t, e_{2}, \psi_{1}\left(t, \zeta_{1}\right)\right) e_{2} \\
\dot{\zeta}_{1} & =F_{\zeta_{1}}\left(t, \zeta_{1}\right) .
\end{aligned}
$$

which, in turn, is also in cascaded form. Now, in view of the structure of $G$, we have

$$
\frac{\partial V_{1}}{\partial e_{i}} G\left(t, e_{i}, \xi_{i}\right) e_{i}=0, \quad \forall i \leq n
$$

hence, the total derivative of $V_{1}$ along the trajectories of (62a) yields

$$
\dot{V}_{1}\left(e_{2}(t)\right) \leq c V_{1}\left(e_{2}(t)\right) \sup _{t \in\left[t_{\circ}, t_{\max }\right)}\left|\eta_{1}(t)\right| \leq c^{\prime} V_{1}\left(e_{2}(t)\right)
$$

with an appropriate redefinition of $c$ and $c^{\prime}-c f$. Ineq. (57). Completeness of $e_{2}(t)$, and therefore of $\eta_{2}(t)$, follows using similar arguments as for the case when $i=1$. Consequently, by Assumption A1, the origin of (62c) is uniformly globally asymptotically stable.

To analyze the stability of the origin for (62) we invoke again [18, Theorem 2]. To that end, we only need to establish uniform global asymptotic stability for the system (63) (since $B$ is linear and the origin of (62c) is uniformly globally asymptotically stable). For this, we invoke [23, Theorem 4] as follows: first, we remark that the respective origins of $\dot{e}_{2}=A_{v_{r}}\left(t, e_{2}\right)$ and (63b) are uniformly globally asymptotically stable. Second, note that condition A4 in [23, Theorem 4] is not needed here since we already established uniform forward completeness. Finally, [23, Ineq. (24)] holds trivially with $V=V_{1}$, in view of (64). We conclude that $\left(e_{2}, \zeta_{1}, \eta_{2}\right)=(0,0,0)$ is a uniformly globally asymptotically stable equilibrium of (62). For $i=3$ the closed-loop dynamics is

$$
\begin{aligned}
& \dot{e}_{3}=A_{v_{r}}\left(t, e_{3}\right) e_{3}+G\left(t, e_{3}, \psi_{2}\left(t, \zeta_{12}\right) e_{3}+B\left(e_{3}\right) \eta_{3}\right. \\
& \dot{\zeta}_{12}=: \quad F_{\zeta_{12}}\left(t, \zeta_{12}\right) \\
& \dot{\eta}_{3}=F_{3_{c l}}\left(t, \tilde{v}_{3}, \tilde{\omega}_{3}, e_{3}(t)\right) \\
& \psi_{2}\left(t, \zeta_{12}\right):=\left[\begin{array}{c}
\tilde{v}_{2}+\left[\xi_{21}+v_{r}(t)\right]\left[\cos \left(e_{\theta 1}\right)+k_{x 1} e_{x 1}-v_{r}\right. \\
\tilde{\omega}_{2}+\xi_{22}+k_{\theta 1} e_{\theta_{1}}+v_{r}(t) k_{y 1} e_{y 1} \phi\left(e_{\theta 1}\right)
\end{array}\right]
\end{aligned}
$$

which corresponds to $\xi_{3}-c f$. (61). The previous arguments, as for the case $i=2$, apply now to (65) so the result follows by induction.

\section{A NUMERICAL EXAMPLE}

We consider a group of four mobile robots modeled as in [24] and following a virtual leader. In this simulation, the desired formation shape of the four mobile robots is a diamond configuration that tracks the trajectory of the virtual leader. See Figure 2. We define the reference velocities such that their sum of squares is persistently exciting, that is,

$$
v_{r}(t)=\sin (0.5 t) M_{v_{r}}(t), \quad \omega_{r}(t)=\sin (0.6 t) M_{\omega_{r}}(t),
$$

where $\left(M_{v_{r}}(t), M_{\omega_{r}}(t)\right)$ are square functions of time of amplitudes $(0.5,0.5)$, periods $(4 \pi, 5 \pi)$, and duty cycles $(1 / 2,100 / 3)$ respectively. The initial conditions are set to $\left[x_{r}(0), y_{r}(0), \theta_{r}(0)\right]$ $=[0,0,0],\left[x_{1}(0), y_{1}(0), \theta_{1}(0)\right]=[1,2,4],\left[x_{2}(0), y_{2}(0), \theta_{2}(0)\right]$ $=[0,2,2],\left[x_{3}(0), y_{3}(0), \theta_{3}(0)\right]=[0,5,1]$ and $\left[x_{4}(0), y_{4}(0), \theta_{4}(0)\right]$ $=[2,2,1]$; the control gains were set to $k_{x_{i}}=k_{y_{i}}=k_{\theta_{i}}=1$, 
and $k_{d}=15$. The formation shape with a certain desired distance between the robots is obtained by setting all desired orientation offsets to zero and defining $\left[d_{x_{r, 1}}, d_{y_{r, 1}}\right]=[0,0],\left[d_{x_{1,2}}, d_{y_{1,2}}\right]=[-1,0]$ and $\left[d_{x_{2,3}}, d_{y_{2,3}}\right]=[1 / 2,-1 / 2]$ and $\left[d_{x_{3,4}}, d_{y_{3,4}}\right]=[0,1]$. The simulation results are showed in Figures $1-2$, in which are depicted the tracking error trajectories between an agent and its neighborhood as well as the physical path described by the swarm.

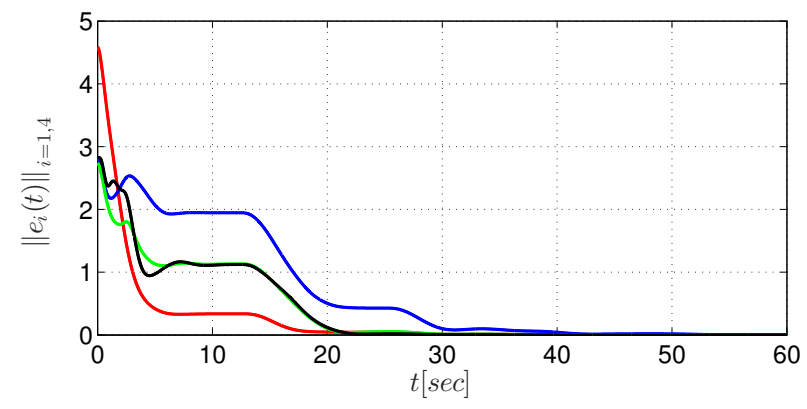

Fig. 1. Exponential convergence of the relative errors (in norm) for each pair leader-follower

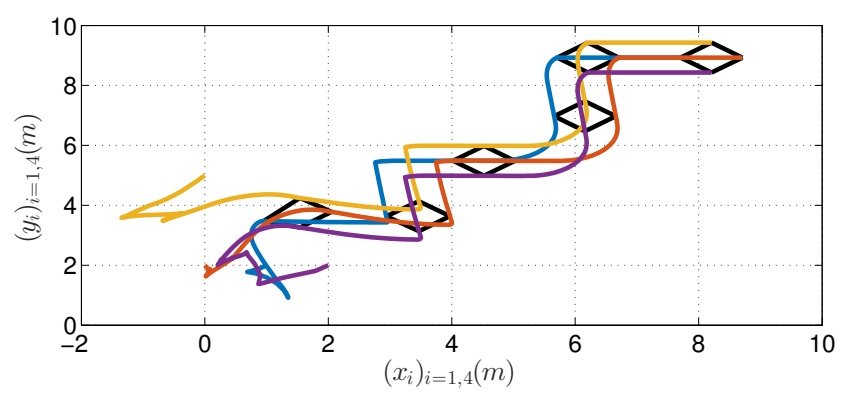

Fig. 2. Illustration of the path-tracking in formation

\section{Conclusions}

A formation-tracking controller for autonomous vehicles that ensures uniform global asymptotic stability of the closed-loop system, under the sole assumption that either the angular or the forward reference velocity is persistently exciting has been presented. Because the tracking problems at the velocity-kinematics and force-dynamics levels are treated separately, the main results apply to any controller at the dynamic level.

Our main contribution lies in providing a strict Lyapunov function for the error dynamics at the kinematics level. This is a fundamental building block to carry on the analysis towards more general cases of interest such as considering parametric uncertainty (adaptive control), output-feedback designs, and collision-avoidance scenarios. Further research in such directions is being carried out.

\section{REFERENCES}

[1] K.-K. Oh, M.-C. Park, and H.-S. Ahn, "A survey of multi-agent formation control," Automatica, vol. 53, pp. 424-440, 2015.

[2] J. Huang, C. Wen, W. Wang, and Z.-P. Jiang, "Adaptive output feedback tracking control of a nonholonomic mobile robot," Automatica, vol. 50, no. 3, pp. 821-831, 2014.
[3] E. Vos, A. J. van der Schaft, and J. M. A. Scherpen, "Formation control and velocity tracking for a group of nonholonomic wheeled robots," IEEE Transactions on Automatic Control, vol. 61, pp. 2702-2707, Sept 2016.

[4] T.-C. Lee, K.-T. Song, C.-H. Lee, and C.-C. Teng, "Tracking control of unicycle-modeled mobile robots using a saturation feedback controller," IEEE Trans. Contr. Syst. Technol., vol. 9, no. 2, pp. 305-318, 2001.

[5] X. Liang, Y. H. Liu, H. Wang, W. Chen, K. Xing, and T. Liu, "Leader-following formation tracking control of mobile robots without direct position measurements," IEEE Transactions on Automatic Control, vol. PP, no. 99, pp. 1-1, 2016.

[6] D. Sun, C. Wang, W. Shang, and G. Feng, "A synchronization approach to trajectory tracking of multiple mobile robots while maintaining timevarying formations," IEEE Transactions on Robotics, vol. 25, pp. 10741086, Oct 2009.

[7] W. Wang, J. Huang, C. Wen, and H. Fan, "Distributed adaptive control for consensus tracking with application to formation control of nonholonomic mobile robots," Automatica, vol. 50, no. 4, pp. 1254 - 1263 , 2014.

[8] A. Dong and J. A. Farrell, "Cooperative control of multiple nonholonomic mobile agents," IEEE Trans. on Automat. Contr., vol. 53, no. 6 , pp. $1434-1447,2008$

[9] S. J. Yoo and T.-H. Kim, "Distributed formation tracking of networked mobile robots under unknown slippage effects," Automatica, vol. 54, pp. 100-106, 2015.

[10] M. Maghenem, A. Loría, and E. Panteley, "Formation-tracking control of autonomous vehicles under relaxed persistency of excitation conditions," IEEE Trans. Contr. Syst. Technol., 2017. DOI: 10.1109/TCST.2017.2734053.

[11] C. C. de Wit, H. Khennouf, C. Samson, and O. J. Sørdalen, ”Nonlinear control design for mobile robots", vol. 11 of Robotics and Automated Systems, ch. Recent Trends in Mobile Robots. Y. F. Zheng, ed., London: World Scientific, 1993.

[12] K. D. Do and J. Pan, "Nonlinear formation control of unicycle-type mobile robots," J. Rob. and Aut. Syst., vol. 55, no. 3, pp. 191-204, 2007.

[13] M. Maghenem, A. Loría and E. Panteley, "Lyapunov-based formationtracking control of nonholonomic systems under persistency of excitation," IFAC-PapersOnLine, vol. 49, no. 18, pp. 404-409, 2016. Presented at IFAC NOLCOS 2016, Monterey, CA, USA.

[14] A. Loría, J. Dasdemir, and N. Alvarez-Jarquin, "Leader-follower formation control of mobile robots on straight paths," IEEE Trans. on Contr. Syst. Techn., vol. 24, no. 2, pp. 727-732, 2016.

[15] E. Panteley, A. Loría, and A. Teel, "Relaxed persistency of excitation for uniform asymptotic stability," IEEE Trans. on Automat. Contr., vol. 46, no. 12 , pp. $1874-1886,2001$.

[16] M. Malisoff and F. Mazenc, Constructions of Strict Lyapunov functions. London: Springer Verlag, 2009.

[17] A. Loría, "From feedback to cascade-interconnected systems: Breaking the loop," in Proc. 47th. IEEE Conf. Decision Contr., (Cancun, Mex.), pp. 4109-4114, 2008.

[18] E. Panteley and A. Loría, "On global uniform asymptotic stability of non linear time-varying non autonomous systems in cascade," Syst. \& Contr. Letters, vol. 33, no. 2, pp. 131-138, 1998.

[19] M. Maghenem, A. Loría, and E. Panteley, "iISS formation tracking control of autonomous vehicles," tech. rep., CentraleSupelec, 2016. Available online: https://hal.archives-ouvertes.fr/hal-01364791.

[20] K. D. Do, "Formation tracking control of unicycle-type mobile robots," in Proceedings 2007 IEEE International Conference on Robotics and Automation, pp. 2391-2396, April 2007.

[21] J. J. Slotine and W. Li, "Adaptive manipulator control: a case study," IEEE Trans. on Automat. Contr., vol. AC-33, pp. 995-1003, 1988.

[22] A. Loría R. Kelly, and A. Teel, "Uniform parametric convergence in the adaptive control of mechanical systems," European J. of Contr., vol. 11, no. 2, pp. 87-100, 2005.

[23] E. Panteley and A. Loría, "Growth rate conditions for stability of cascaded time-varying systems," Automatica, vol. 37, no. 3, pp. 453460, 2001

[24] K. D. Do, "Formation tracking control of unicycle-type mobile robots with limited sensing ranges," IEEE Trans. Contr. Syst. Technol., vol. 16, no. 3, pp. 527-538, 2008. 